\title{
PET Imaging of Glutaminolysis in Tumors by ${ }^{18}$ F-(2S,4R)4-Fluoroglutamine
}

\author{
Brian P. Lieberman ${ }^{1}$, Karl Ploessl ${ }^{1}$, Limin Wang ${ }^{1}$, Wenchao $\mathrm{Qu}^{1}$, Zhihao $\mathrm{Zha}^{1}$, David R. Wise ${ }^{2}$, Lewis A. Chodosh ${ }^{2}$, \\ George Belka ${ }^{2}$, Craig B. Thompson ${ }^{2}$, and Hank F. Kung ${ }^{1,3}$ \\ ${ }^{1}$ Department of Radiology, University of Pennsylvania, Philadelphia, Pennsylvania; ${ }^{2}$ Department of Cancer Biology, Abramson \\ Cancer Center, University of Pennsylvania, Philadelphia, Pennsylvania; and ${ }^{3}$ Department of Pharmacology, University of \\ Pennsylvania, Philadelphia, Pennsylvania
}

\begin{abstract}
Changes in gene expression, metabolism, and energy requirements are hallmarks of cancer growth and self-sufficiency. Upregulation of the PI3K/Akt/mTor pathway in tumor cells has been shown to stimulate aerobic glycolysis, which has enabled ${ }^{18} \mathrm{~F}-\mathrm{FDG}$ PET tumor imaging. However, of the millions of ${ }^{18} \mathrm{~F}$-FDG PET scans conducted per year, a significant number of malignant tumors are ${ }^{18}$ F-FDG PET-negative. Recent studies suggest that several tumors may use glutamine as the key nutrient for survival. As an alternative metabolic tracer for tumors, ${ }^{18} \mathrm{~F}-(2 S, 4 R) 4$-fluoroglutamine was developed as a PET tracer for mapping glutaminolytic tumors. Methods: A series of in vitro cell uptake and in vivo animal studies were performed to demonstrate tumor cell addiction to glutamine. Cell uptake studies of this tracer were performed in SF188 and 9L glioblastoma tumor cells. Dynamic small-animal PET studies of ${ }^{18} \mathrm{~F}-$ $(2 S, 4 R) 4$-fluoroglutamine were conducted in 2 animal models: xenografts produced in F344 rats by subcutaneous injection of 9L tumor cells and transgenic mice with M/tomND spontaneous mammary gland tumors. Results: In vitro studies showed that both transformed 9L and SF188 tumor cells displayed a high rate of glutamine uptake (maximum uptake, $\approx 16 \%$ dose/100 $\mu \mathrm{g}$ of protein). The cell uptake of ${ }^{18} \mathrm{~F}-(2 S, 4 R) 4$-fluoroglutamine by SF188 cells is comparable to that of ${ }^{3} \mathrm{H}$-L-glutamine but higher than that of ${ }^{18} \mathrm{~F}-\mathrm{FDG}$. The tumor cell uptake can be selectively blocked. Biodistribution and PET studies showed that ${ }^{18} \mathrm{~F}-(2 S, 4 R) 4-$ fluoroglutamine localized in tumors with a higher uptake than in surrounding muscle and liver tissues. Data suggest that certain tumor cells may use glutamine for energy production. Conclusion: The results support that ${ }^{18} \mathrm{~F}$ $(2 S, 4 R) 4$-fluoroglutamine is selectively taken up and trapped by tumor cells. It may be useful as a novel metabolic tracer for tumor imaging.
\end{abstract}

Key Words: PET; tumor metabolism; ${ }^{18}$ F-FDG; gene expression; c-Myc

J Nucl Med 2011; 52:1947-1955

DOI: 10.2967/jnumed.111.093815

\footnotetext{
Received May 27, 2011; revision accepted Jul. 29, 2011.

For correspondence or reprints contact: Hank F. Kung, Departments of Radiology and Pharmacology, University of Pennsylvania, 3700 Market St., Philadelphia, PA 19104.

E-mail: kunghf@sunmac.spect.upenn.edu

Published online Nov. 15, 2011.

COPYRIGHT @ 2011 by the Society of Nuclear Medicine, Inc.
}

$\mathbf{C}$ hanges of cellular metabolism in tumor cells are prerequisites for the proliferation and uncontrolled growth associated with cancer. Indeed, Otto Warburg first described aerobic glycolysis in cancer cells in 1924 (1-3). Aerobic glycolysis describes the phenomenon in which tumors cells use glycolysis to produce energy in an oxygen-rich environment. Instead of solely serving as a source of cellular energy, adenosine triphosphate, the mitochondrial tricarboxylic acid (TCA) cycle is diverted to make metabolic intermediates for fatty acid and amino acid synthesis needed for tumor growth and survival. These metabolic changes appear to offer advantages for the survival and proliferation of the tumor. In tumor cells, there is now a widespread acceptance that the $\mathrm{PI} 3 \mathrm{~K} / \mathrm{Akt} / \mathrm{mTOR}$ pathway upregulates aerobic glycolysis and supports tumor sustainability (Fig. 1). There is strong evidence that increased glucose utilization in tumor cells is linked to increased glucose transporters and hexokinase II enzymes, which is the biochemical basis for using ${ }^{18} \mathrm{~F}-\mathrm{FDG}$ PET for the detection and monitoring of malignant tumors in cancer patients $(4,5)$. Every year, millions of ${ }^{18} \mathrm{~F}$-FDG scans are conducted within the United States. Despite the tremendous promise and use of ${ }^{18} \mathrm{~F}$-FDG PET, a noticeable portion of these tumors, which are ${ }^{18}$ F-FDG PET-negative, go undetected (6), possibly suggesting that some types of tumors are switching their metabolism and energy consumption from glucose to other nutrients, such as glutamine. Glutamine has the highest concentration $(0.5-1 \mathrm{mM})$ among all of the amino acids circulating in the blood. When the oncogene c-Myc is upregulated, the tumor cells may turn to glutamine as its major energy source, using the TCA cycle within the mitochondria to produce energy and cell building blocks (7). This may be an essential strategy adopted by cancer cells for growth and survival. In addition, the tumor cells may likely use both pathways, PI3K/Akt/mTOR and c-Myc pathways, to generate energy for growth and survival. These processes may not be mutually exclusive and therefore, this may be a clever and essential strategy adopted by tumor cells for survival $(2,8-11)$. Recently, a series of papers suggests that ${ }^{18} \mathrm{~F}-\mathrm{FDG}$-negative tumors may use a different metabolic pathway-glutaminolysis $(7,12-16)$. The results, at least 
partially, provide a probable explanation for the observation that ${ }^{18} \mathrm{~F}-\mathrm{FDG}$ PET fails to spot certain tumors in cancer patients. We, therefore, have hypothesized a novel series of metabolic tracers for PET based on the glutamine addiction of tumor cells. We use the c-Myc-amplified glioblastoma cell line SF188 as a model of glutamine-addicted tumor (7).

Recently, chemical synthesis and characterization of 4 isomers of 4-fluoroglutamine have been reported (17). One of the isomers, ${ }^{18} \mathrm{~F}-(2 S, 4 R) 4$-fluoroglutamine, was fully identified, and a detailed radiochemistry preparation was successfully implemented. This tracer appeared to be a suitable candidate, showing high tumor cell uptake and retention (17). Reported herein are results of in vitro and in vivo evaluations of this novel metabolic tracer, an optically pure ${ }^{18} \mathrm{~F}-(2 S, 4 R) 4$-fluoroglutamine, for PET of glutamine-addicted tumors.

\section{MATERIALS AND METHODS}

All chemical reagents used were obtained and used directly unless otherwise stated. All procedures in this study followed the guidelines of the Institutional Animal Care and Use Committee of University of Pennsylvania.

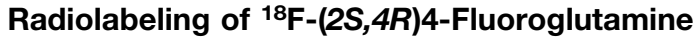

The radiosynthesis was performed by a method as described before (17). The supplemental information (available online only at http://jnm.snmjournals.org) provides detailed procedures. The radiochemical and stereochemical purity of the final product was determined by chiral high-performance liquid chromatography (HPLC) (Chirex 3126 (d)-penicillamine, $1 \mathrm{mM} \mathrm{CuSO}_{4}$ solution, $1 \mathrm{~mL} / \mathrm{min}$; retention time for the $(2 S, 4 R)$ isomer, $\sim 11 \mathrm{~min}$, and the $(2 R, 4 R)$ isomer, $\sim 18 \mathrm{~min}$ ) (radiochemical yield [non-decaycorrected], $8.4 \% \pm 3.4 \%$; radiochemical purity, $98 \% \pm 1 \%$; optical purity, $>91 \% \pm 8 \% ; n=10$ ).

\section{Cell Culture, In Vitro Cell Uptake, Protein Incorporation, and Inhibition Studies}

Detailed procedures for using 9L cells, SF188 cells, and Bcl$\mathrm{xL}$-transfected $\mathrm{SF} 188_{\text {bcl-xl }}$ for studying ${ }^{18} \mathrm{~F}-(2 S, 4 R) 4$-fluoroglutamine are included in the supplemental information.

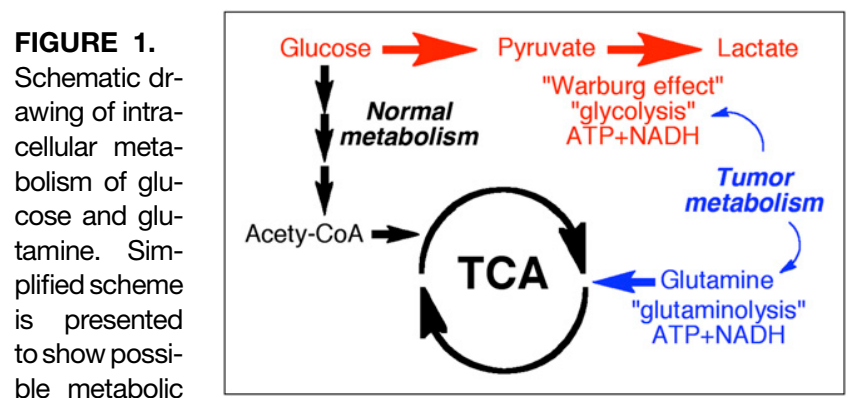

ble metabolic

changes in

tumor cells using glycolysis (red) or glutaminolysis (blue). Tumor cells may use both pathways to generate energy and intermediate metabolites for survival and growth. However, tumor cells may switch energy source to glutamine and, thus, enhance their proliferation. Acety-CoA = acetyl coenzyme $\mathrm{A} ; \mathrm{ATP}=$ adenosine triphosphate; $\mathrm{NADH}=$ reduced nicotinamide adenine dinucleotide.

\section{In Vivo Biodistribution Study in Normal Institute of Cancer Research (ICR) Male Mice and in Fischer 344 Rats Bearing 9L Tumors}

Biodistribution procedures for ${ }^{18} \mathrm{~F}-(2 S, 4 R) 4$-fluoroglutamine in normal ICR mice and Fischer 344 rats bearing 9L tumors are included in the supplemental information.

\section{Small-Animal PET Imaging}

Dynamic small-animal PET imaging studies were conducted with ${ }^{18} \mathrm{~F}-(2 S, 4 R) 4$-fluoroglutamine on a dedicated animal PET scanner (Mosaic; Phillips) that has a field of view of $11.5 \mathrm{~cm}$. F344 rats with 9L xenografts and transgenic mice bearing M/ tomND spontaneous human mammary tumors were used for the imaging studies. There are many advantages for using this transgenic mouse model. These mice are genetically engineered with a doxycycline-sensitive promoter driving myc gene expression. Compared with traditional xenograft tumor models, these tumors spontaneously arise. When the mice are administered doxycycline through their drinking water $(2 \mathrm{mg} / \mathrm{mL})$, the expression of the myc gene is upregulated. This model was chosen because these compounds will allow a direct correlation between true glutamine uptake and the level of $m y c$ gene expression. All animals were subjected to isoflurane anesthesia $(2 \%-3 \%, 1 \mathrm{~L} / \mathrm{min}$ oxygen) and were then placed on a heating pad to maintain body temperature throughout the procedure. Animals were visually monitored for breathing and any other signs of distress throughout the entire imaging period. F344 rats were injected with $19-37 \mathrm{MBq}$ and transgenic mice were injected with $7.4-15 \mathrm{MBq}$ of ${ }^{18} \mathrm{~F}-(2 S, 4 R)$ 4-fluoroglutamine, respectively. Data acquisition began after an intravenous injection of the tracer. All scans were conducted over a period of $2 \mathrm{~h}$ (dynamic, $5 \mathrm{~min} /$ frame). The frames were analyzed and reconstructed with AMIDE's a Medical Imaging Data Examiner imaging analysis software.

\section{Ex Vivo PET Study in Transgenic Mice with M/tomND Spontaneous Tumors}

${ }^{18} \mathrm{~F}-(2 S, 4 R) 4$-fluoroglutamine (37 MBq) was injected intravenously while the mouse was subjected to isoflurane anesthesia (2\%-3\%, $1 \mathrm{~L} / \mathrm{min}$ oxygen). The mouse was sacrificed $30 \mathrm{~min}$ after injection, and the organs of interest were removed and placed on a $9 \times 11 \mathrm{~cm}$ cardboard cut-out. The cut-out containing the organs was then placed within the animal PET scanner (Mosaic; Phillips) and imaging commenced. A 5-min scan was obtained. Data were analyzed and reconstructed with AMIDE.

\section{In Vivo Metabolism Studies in Transgenic Mice with m/tomND Spontaneous Tumors and F344 Rats Bearing 9L Tumor Xenografts}

Approximately $11-19 \mathrm{MBq}$ of ${ }^{18} \mathrm{~F}-(2 S, 4 R) 4$-fluoroglutamine were injected intravenously while the rodents were under isoflurane anesthesia $(2 \%-3 \%, 1 \mathrm{~L} / \mathrm{min}$ oxygen). The rodents were sacrificed $30 \mathrm{~min}$ after injection, and the tumors were removed (3 separate $\mathrm{m} /$ tomND tumors were removed from 3 separate mice, and $29 \mathrm{~L}$ tumors were processed from F344 rats). Tumors were separately homogenized in $1 \mathrm{~mL}$ of phosphate-buffered saline (containing $0.90 \mathrm{mM}$ of $\mathrm{Ca}^{2+}$ and $1.05 \mathrm{mM}$ of $\mathrm{Mg}^{2+}$ ) with a Wheaton overhead stirrer and centrifuged at 18,000g for $6 \mathrm{~min}$. Layers were separated and counted with a $\gamma$-counter (Cobra II; Packard) (0.5 min/sample, $80 \%$ efficiency). Supernatant was filtered through a $0.45-\mu \mathrm{m}$ syringe filter. HPLC analysis was then conducted on the supernatant sample: the sample $(105 \mu \mathrm{L})$ in an HPLC vial was counted in a $\gamma$-counter, and $100 \mu \mathrm{L}$ were injected 


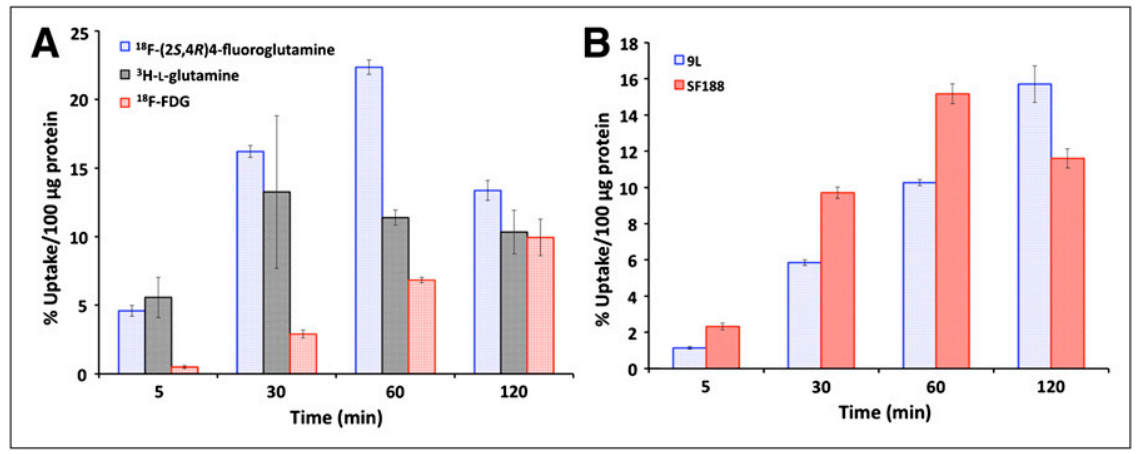

FIGURE 2. (A) In vitro uptake studies of ${ }^{18} \mathrm{~F}-(2 \mathrm{~S}, 4 R) 4$-fluoroglutamine (blue) in SF188 $8_{\text {bcl-xL }}$ cell line. ${ }^{3} \mathrm{H}-\mathrm{L}-$ glutamine (black) and ${ }^{18} \mathrm{~F}-\mathrm{FDG}$ (red) were used as reference ligands. (Reprinted with permission of (17).) (B) Uptake of ${ }^{18} \mathrm{~F}-(2 \mathrm{~S}, 4 R) 4$-fluoroglutamine in $9 \mathrm{~L}$ and SF188 cells. Values are represented as mean $\pm \mathrm{SD}, n=3$. onto HPLC. The vial was recounted in the $\gamma$-counter, and the injected activity was determined. HPLC samples were collected in 1-min fractions in an automated fraction collector for $60 \mathrm{~min}$. The samples were counted in a $\gamma$-counter for $0.5 \mathrm{~min}$. The counts were decay-corrected to injection time and added up. More than $90 \%$ of the counts were recovered from HPLC. The counts were plotted intensity (cpm) versus fractions to show the profile of the sample. The relative retention times were compared with cold sample injected under similar conditions $(100-\mu \mathrm{L}$ injections) to determine the retention times of ${ }^{18} \mathrm{~F}-(2 S, 4 R) 4$-fluoroglutamine and ${ }^{18} \mathrm{~F}-(2 S, 4 R) 4$-fluoroglutamic acid and ${ }^{18} \mathrm{~F}$-fluoride. Area under the curves for the correct retention times for ${ }^{18} \mathrm{~F}$-fluoride, ${ }^{18} \mathrm{~F}-(2 S, 4 R)$ 4-fluoroglutamine, and ${ }^{18} \mathrm{~F}-(2 S, 4 R)$ 4-fluoroglutaminic acid were determined.

\section{RESULTS}

\section{In Vitro Cell Uptake Studies}

Time-dependent uptake of ${ }^{18} \mathrm{~F}-(2 S, 4 R) 4$-fluoroglutamine in phosphate-buffered saline was determined in rat glioma 9L, a commonly used proliferative cell line for evaluation of tumor imaging agents $(18,19)$. A second type of tumor cell line, SF188, derived from human glioblastoma, displays a 25-fold amplification of oncogene c-Myc (7). It has been demonstrated that elevated levels of c-Myc lead to glutamine addiction and increases uptake of glutamine. Results in Figure 2 demonstrated high cell uptake of ${ }^{18} \mathrm{~F}-$ $(2 S, 4 R) 4$-fluoroglutamine in both tumor cell lines. In 9L cells, this new tracer showed a linear increasing uptake and reached a maximum of $15.7 \pm 1.0$ percentage injected dose (\%ID) per $100 \mu \mathrm{g}$ of protein at $120 \mathrm{~min}$. This value is comparable to or a little higher than that reported for ${ }^{18} \mathrm{~F}$ FACBC (1-amino-3- ${ }^{18}$ F-fluorocyclobutanecarboxylic acid), another ${ }^{18} \mathrm{~F}$-labeled amino acid for tumor imaging developed under a totally different uptake mechanism $(18,20,21)$. In SF188 cells, uptake of ${ }^{18} \mathrm{~F}-(2 S, 4 R) 4$-fluoroglutamine exhibited different kinetics from those in $9 \mathrm{~L}$ cells and reached maximum around $60 \mathrm{~min}$ and then decreased. Within the first $60 \mathrm{~min}$, the rate of the uptake was higher in SF188 cells, and the uptake value in SF188 at 60 min was 1.5 -fold higher than the corresponding uptake in $9 \mathrm{~L}$ cells.

We also compared the cell uptake of ${ }^{18} \mathrm{~F}-(2 S, 4 R) 4$-fluoroglutamine to ${ }^{18} \mathrm{~F}-\mathrm{FDG}$ in SF188 using ${ }^{3} \mathrm{H}$-L-glutamine as the internal reference (Fig. 2). Results indicate that the uptake of ${ }^{18} \mathrm{~F}-(2 S, 4 R) 4$-fluoroglutamine was significantly higher than that of ${ }^{18} \mathrm{~F}-\mathrm{FDG}$. The maximum uptake of ${ }^{18} \mathrm{~F}-(2 S, 4 R) 4$-fluoroglutamine was more than 2-fold higher than that of ${ }^{18} \mathrm{~F}-\mathrm{FDG}$. Because the experiments were performed under the same condition using 2 tracers at the same time, the results of this dual-isotope experiments strongly suggest that the cell uptake of this new tracer, ${ }^{18} \mathrm{~F}-(2 S, 4 R) 4-$ fluoroglutamine, is likely associated with the upregulation of c-Myc in SF188 cells.

\section{Inhibition of ${ }^{18} \mathrm{~F}-(2 \mathrm{~S}, 4 R) 4-\mathrm{Fluoroglutamine}$ Cell Uptake}

To investigate the transport mechanisms involved in the uptake of ${ }^{18} \mathrm{~F}-(2 S, 4 R) 4$-fluoroglutamine, we have conducted a series of competitive inhibition studies in 9L cells using specific inhibitors for system A, ASC, L, and N, which are major amino acid transport systems in mammalian cells and are potentially responsible for uptake of glutamine and its analogs $(22,23)$. The result showed that system A inhibitor methylaminoisobutyric acid had no inhibitory effect on the uptake of ${ }^{18} \mathrm{~F}-(2 S, 4 R) 4$-fluoroglutamine (Supplemental Fig. 1), suggesting that system A amino acid transport was not involved in the uptake of this new tracer. System L inhibitor BCH, system ASC inhibitor L-serine, and system ASC N inhibitor L-glutamine exhibited

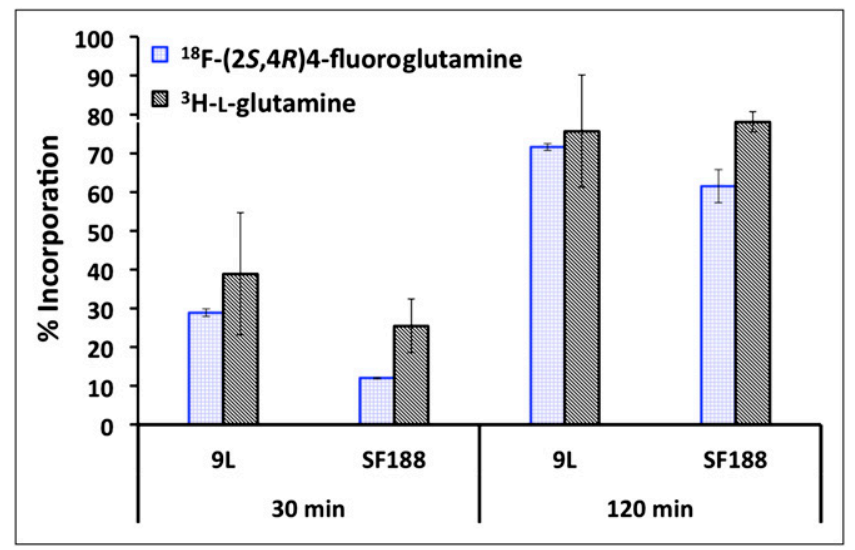

FIGURE 3. Incorporation of ${ }^{18} \mathrm{~F}-(2 \mathrm{~S}, 4 R) 4$-fluoroglutamine and ${ }^{3} \mathrm{H}$ L-glutamine into protein in $9 \mathrm{~L}$ and SF188 tumor cells. Comparison of cellular uptake of ${ }^{18} \mathrm{~F}$ - $(2 \mathrm{~S}, 4 R) 4$-fluoroglutamine and ${ }^{3} \mathrm{H}$-L-glutamine was performed using dual-isotope experiments at $30-$ and 120-min incubation times. 
FIGURE 4. (Upper) Small-animal PET images of ${ }^{18} \mathrm{~F}-(2 S, 4 R) 4$-fluoroglutamine in F344 9L rat after intravenous injection. Data represent images from summed 2-h scan. Images are shown in transverse, coronal, and sagittal views. Arrows represent location of tumors. (Lower) Small-animal PET time-activity curve for ${ }^{18} \mathrm{~F}-(2 S, 4 R) 4$-fluoroglutamine after intravenous injection into F344 rat bearing a xenografted 9L tumor on left shoulder.

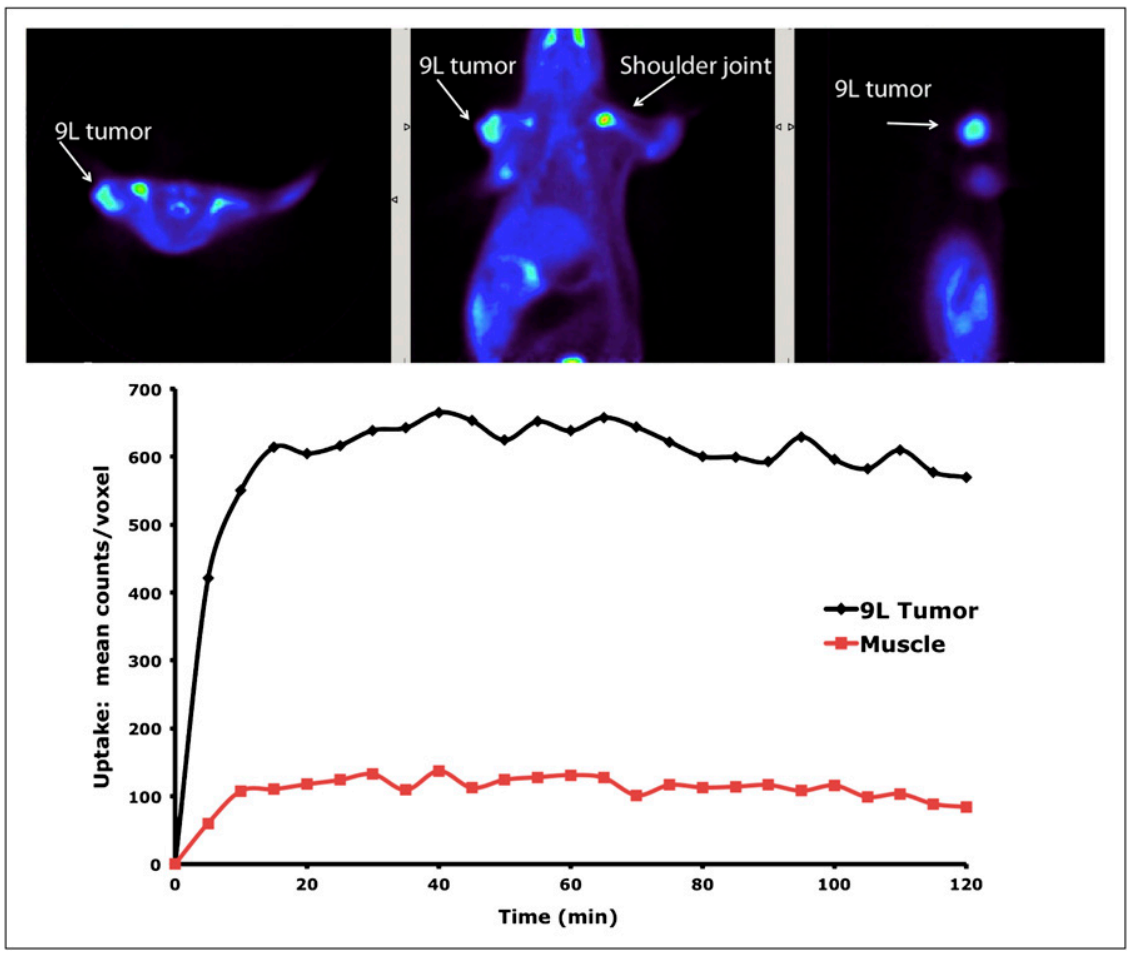

a similar concentration-dependent reduction of cell uptake, thus indicating potential involvement of systems L, ASC, and $\mathrm{N}$ in the uptake of ${ }^{18} \mathrm{~F}-(2 S, 4 R) 4$-fluoroglutamine.

Further examination of transport mechanisms by $\mathrm{Na}^{+}$ and $\mathrm{pH}$ dependence studies showed that the uptake of ${ }^{18} \mathrm{~F}-(2 S, 4 R) 4$-fluoroglutamine was reduced by $78 \%$ in $\mathrm{Na}^{+}$-free medium and was insensitive to $\mathrm{pH}$ change from 6 to 8 (Supplemental Fig. 1). The activity of system $\mathrm{N}$ is highly $\mathrm{pH}$-sensitive and is almost inactive in $\mathrm{pH} 6$ (24), possibly suggesting that system $\mathrm{N}$ did not play an important role in the transport. Moreover, system ASC is sodiumdependent, whereas system $\mathrm{L}$ is sodium-independent (25). These results indicate that system $\mathrm{L}$ might be responsible for sodium-independent uptake of ${ }^{18} \mathrm{~F}-(2 S, 4 R) 4$-fluoroglu- tamine whereas sodium-dependent uptake of the tracer was predominate through system ASC. We also differentiated the roles of 2 subtypes of amino acid transport systems (ASC: ASCT1 and ASCT2) (26) in the uptake of ${ }^{18} \mathrm{~F}$ $(2 S, 4 R) 4$-fluoroglutamine. ASCT2 is overexpressed in a variety of cancer cells and is important for tumor growth and survival $(27,28)$. To examine the selectivity of ${ }^{18} \mathrm{~F}$ $(2 S, 4 R) 4$-fluoroglutamine toward ASCT2, we compared the inhibitory effect of L- $\gamma$-glutamyl-p-nitroanilide (GPNA), a potent ASCT2 inhibitor (29), to its close analog L- $\gamma$-glutamyl-anilide, which is inactive toward ASCT2 (Supplemental Fig. 1). GPNA had more impact on the uptake: $1 \mathrm{mM}$ GPNA could reduce the uptake by $77 \%$, compared with $44 \%$, when $1 \mathrm{mM} \mathrm{L}-\gamma$-glutamyl-anilide

TABLE 1

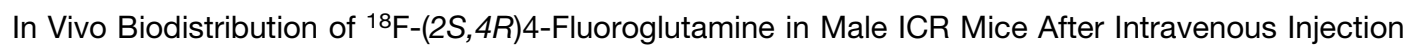

\begin{tabular}{lccccc}
\hline Organ & $2 \mathrm{~min}$ & $30 \mathrm{~min}$ & $60 \mathrm{~min}$ & $120 \mathrm{~min}$ & $240 \mathrm{~min}$ \\
\hline Blood & $6.19 \pm 0.83$ & $2.72 \pm 0.17$ & $2.05 \pm 0.16$ & $0.83 \pm 0.12$ & $0.48 \pm 0.06$ \\
\hline Heart & $4.31 \pm 0.32$ & $3.16 \pm 0.25$ & $2.86 \pm 0.47$ & $1.86 \pm 0.24$ & $1.24 \pm 0.19$ \\
Muscle & $1.62 \pm 0.10$ & $2.48 \pm 0.16$ & $2.86 \pm 0.34$ & $1.81 \pm 0.26$ & $0.94 \pm 0.15$ \\
Lung & $7.15 \pm 0.76$ & $5.36 \pm 0.49$ & $4.37 \pm 0.28$ & $1.69 \pm 0.10$ & $1.03 \pm 0.15$ \\
Kidney & $16.1 \pm 1.03$ & $9.93 \pm 0.52$ & $7.60 \pm 0.84$ & $2.15 \pm 0.34$ & $1.25 \pm 0.21$ \\
Pancreas & $17.2 \pm 1.00$ & $19.7 \pm 2.16$ & $17.5 \pm 2.28$ & $9.57 \pm 1.22$ & $5.92 \pm 0.87$ \\
Spleen & $7.51 \pm 0.44$ & $5.39 \pm 0.72$ & $4.22 \pm 0.38$ & $2.02 \pm 0.16$ & $1.15 \pm 0.14$ \\
Liver & $6.92 \pm 0.92$ & $6.23 \pm 0.50$ & $5.70 \pm 0.62$ & $2.46 \pm 0.26$ & $1.30 \pm 0.18$ \\
Skin & $2.72 \pm 0.05$ & $4.01 \pm 0.24$ & $2.94 \pm 0.68$ & $2.17 \pm 0.16$ & $1.25 \pm 0.13$ \\
Brain & $0.54 \pm 0.05$ & $0.51 \pm 0.05$ & $0.53 \pm 0.07$ & $0.57 \pm 0.05$ & $0.45 \pm 0.06$ \\
Bone & $3.93 \pm 0.37$ & $5.64 \pm 0.69$ & $7.93 \pm 1.02$ & $14.4 \pm 1.31$ & $19.4 \pm 0.50$
\end{tabular}

Data represented as \%ID/g, mean \pm SD (5 mice per time point). 
TABLE 2

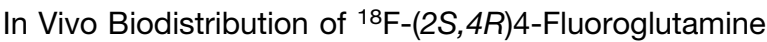
in F344 Rats Bearing 9L Tumor Xenografts After Intravenous Injection

\begin{tabular}{|lcc}
\hline \multicolumn{1}{c}{ Organ } & $30 \mathrm{~min}$ & $60 \mathrm{~min}$ \\
\hline Blood & $0.43 \pm 0.01$ & $0.32 \pm 0.02$ \\
\hline Heart & $0.36 \pm 0.02$ & $0.35 \pm 0.01$ \\
\hline Muscle & $0.37 \pm 0.02$ & $0.38 \pm 0.03$ \\
\hline Lung & $0.64 \pm 0.02$ & $0.41 \pm 0.04$ \\
\hline Kidney & $1.02 \pm 0.12$ & $0.76 \pm 0.18$ \\
\hline Pancreas & $2.14 \pm 0.27$ & $1.36 \pm 0.16$ \\
Spleen & $0.76 \pm 0.05$ & $0.53 \pm 0.04$ \\
\hline Liver & $0.98 \pm 0.15$ & $0.66 \pm 0.13$ \\
Skin & $0.42 \pm 0.11$ & $0.29 \pm 0.04$ \\
\hline Brain & $0.11 \pm 0.01$ & $0.13 \pm 0.00$ \\
\hline Bone & $0.78 \pm 0.13$ & $1.03 \pm 0.38$ \\
\hline Tumor 9L $(n=5)$ & $1.03 \pm 0.14$ & $0.76 \pm 0.21$ \\
\hline Ratio & & \\
\hline Tumor to blood & 2.39 & 2.37 \\
\hline Tumor to muscle & 2.78 & 2.00 \\
\hline
\end{tabular}

Data represented as $\% \mathrm{ID} / \mathrm{g}$, mean $\pm \mathrm{SD}$ (6 rats per time point).

was present. The result suggests that the transport of ${ }^{18} \mathrm{~F}-$ $(2 S, 4 R) 4$-fluoroglutamine might prefer ASCT2 to ASCT1. Results of the transport studies suggest that the transport of ${ }^{18} \mathrm{~F}-(2 S, 4 R) 4$-fluoroglutamine may be predominantly through system ASC and may prefer its subtype ASCT2 (Supplemental Fig. 1).

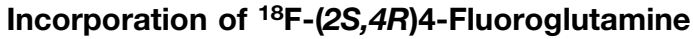 into Protein}

Incorporation of ${ }^{18} \mathrm{~F}-(2 S, 4 R) 4$-fluoroglutamine into protein at 30 and 120 min in 9L and SF188 cells was measured
(Fig. 3). ${ }^{3} \mathrm{H}-\mathrm{L}-$ glutamine, the native glutamine, was used as the reference ligand in this experiment. ${ }^{18} \mathrm{~F}-(2 S, 4 R) 4$-fluoroglutamine demonstrated an incorporation profile similar to the one observed for ${ }^{3} \mathrm{H}$-L-glutamine. A significant percentage of ${ }^{18} \mathrm{~F}-(2 S, 4 R) 4$-fluoroglutamine was incorporated into protein after $2 \mathrm{~h}$. At 30 and $120 \mathrm{~min}$, the protein incorporation of ${ }^{18} \mathrm{~F}-(2 S, 4 R) 4$-fluoroglutamine in $9 \mathrm{~L}$ cells was $29 \%$ and $72 \%$ and $12 \%$ and $62 \%$ in SF188 cells, respectively (Fig. 4).

\section{In Vivo Biodistribution in ICR Mice}

In vivo biodistribution of ${ }^{18} \mathrm{~F}-(2 S, 4 R) 4$-fluoroglutamine in mice showed the expected behavior of a radiolabeled amino acid with significant pancreas uptake (19.7\% dose/g at $30 \mathrm{~min}$ ), most likely due to the exocrine function and high protein turnover within the pancreas. Pancreatic function requires the use of various amino acids as precursors for protein and peptide synthesis. Blood levels drop fairly quickly with time, showing low blood activity at $240 \mathrm{~min}$ after injection $(0.48 \%$ dose/g). There was no significant uptake within the lung, along with no significant retention within the first arteriovenous capillary bed, suggesting that ${ }^{18} \mathrm{~F}-(2 S, 4 R) 4$-fluoroglutamine is fairly hydrophilic and water-soluble. Rapid uptake is observed within the kidneys but is quickly excreted through the urinary bladder. ${ }^{18} \mathrm{~F}-$ $(2 S, 4 R) 4$-fluoroglutamine showed a moderate liver uptake with a relatively slow washout rate. Brain uptake exhibited a relatively low, but consistent, uptake of $0.54 \% \mathrm{ID} / \mathrm{g}$ at 2 min after injection and remained consistent with little to no washout throughout the 240-min experiment. Lastly, bone (femur) showed rapid uptake and increased with time, implying that in vivo defluorination may be occurring after injection (Table 1).

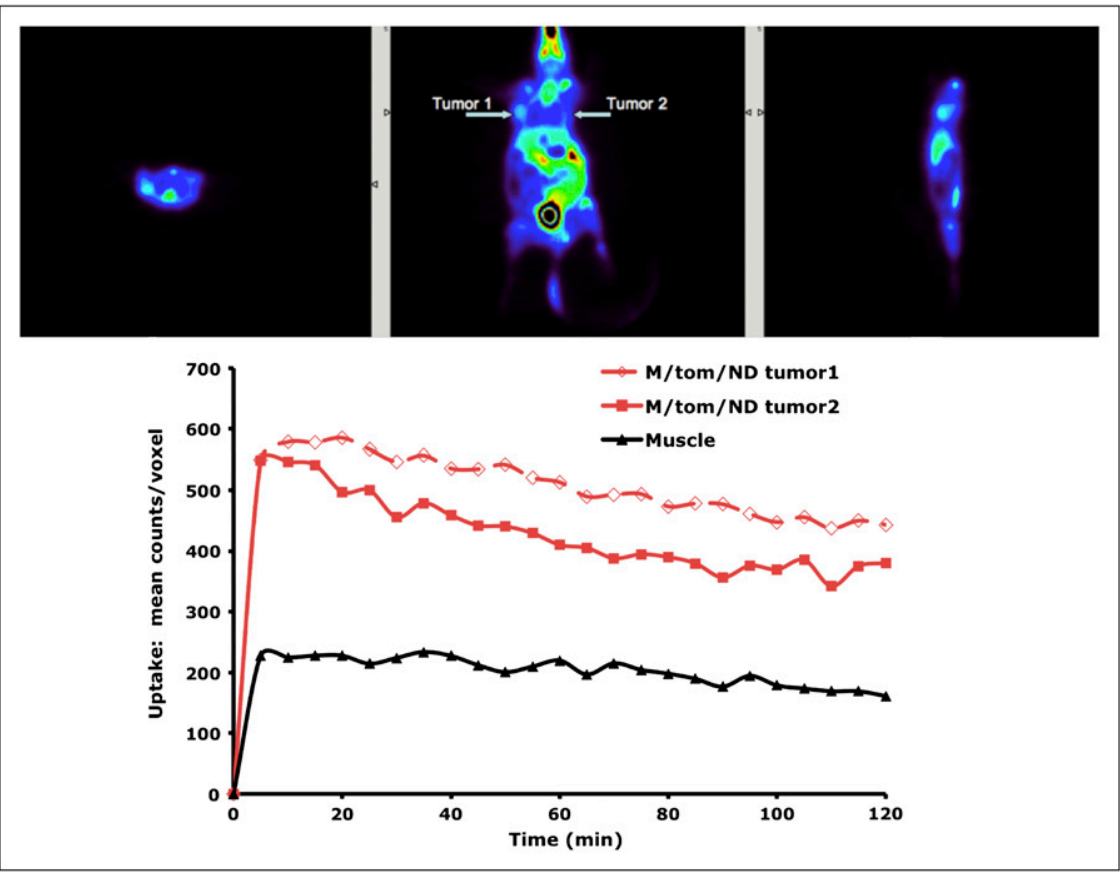

FIGURE 5. (Upper) Small-animal PET images of ${ }^{18} \mathrm{~F}-(2 \mathrm{~S}, 4 R) 4$-fluoroglutamine in transgenic mouse bearing $\mathrm{m} /$ tomND tumors (myc gene upregulated) after intravenous injection. Data represent images from summed 2-h scan. Images are shown in transverse, coronal, and sagittal views. Arrows represent location of tumors. (Lower) Small-animal PET time-activity curve for ${ }^{18} \mathrm{~F}-(2 S, 4 R) 4$-fluoroglutamine after intravenous injection into mouse. 


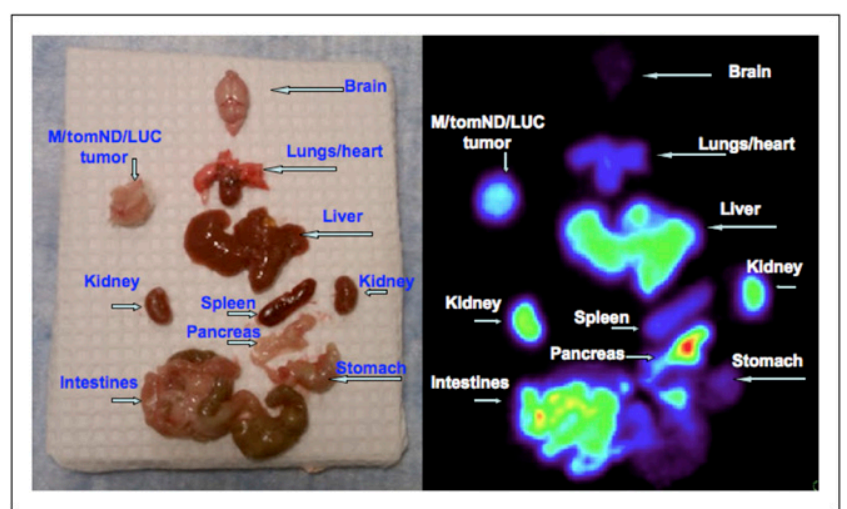

FIGURE 6. Ex vivo animal PET images of genetically modified mouse bearing $\mathrm{m} /$ tomND tumor was evaluated. Tomographic images (sum of $5 \mathrm{~min}$ ) obtained by PET were comparable to planar photograph of organs.

\section{In Vivo Biodistribution in Fischer 344 Rats Bearing 9L Xenografts}

The use and production of 9L xenografts in F344 rats is a well-known and established animal model that represents typical glioblastomas that would be found within a clinical setting $(18,30)$. Uptake of ${ }^{18} \mathrm{~F}-(2 S, 4 R) 4$-fluoroglutamine showed respectable 9L tumor uptake values (uptake of $1.03 \% \mathrm{ID} / \mathrm{g}$ at $30 \mathrm{~min}$ after injection). The 9L tumorto-muscle ratio (target-to-background) decreased slightly from 2.78 at $30 \mathrm{~min}$ to a ratio of 2.00 at $60 \mathrm{~min}$ after injection. Results of biodistribution studies also showed a high pancreas uptake, again due to the various amino acid precursors used for protein and peptide synthesis. Bone uptake (femur) rose slightly from $0.78 \% \mathrm{ID} / \mathrm{g}$ at $30 \mathrm{~min}$ to $1.03 \% \mathrm{ID} / \mathrm{g}$ at $60 \mathrm{~min}$ after injection, suggesting that in vivo defluorination of the compound may be occurring (Table 2).
Animal PET Studies in F344 Rats with 9L Xenografts and Transgenic Mice with M/tomND

Spontaneous Tumors

Dynamic small-animal PET studies on rats bearing 9L tumor xenografts and transgenic mice bearing spontaneous $\mathrm{M} /$ tomND tumors were performed with ${ }^{18} \mathrm{~F}-(2 S, 4 R) 4$-fluoroglutamine. Animal PET images of summed 2-h coronal sections were selected for visualization. As the images demonstrate, clear tumor uptake is visualized within each animal model. To confirm this, region-of-interest analysis was performed using AMIDE analysis software on the reconstructed images to generate the time-activity curves for ${ }^{18} \mathrm{~F}-(2 S, 4 R) 4$-fluoroglutamine. The kinetics indeed confirm that the tracer exhibited higher tumor uptake than muscle (background) region uptake. Rapid tumor uptake is visualized within each animal model within the first $20 \mathrm{~min}$. Tumor uptake remains rather consistent throughout the 2-h scan time, with a slow washout rate observed. High kidney and bladder uptake was observed (Figs. 4 and 5).

Ex vivo animal PET studies in a transgenic mouse with M/tomND spontaneous tumors were also performed after dissection. A clear tumor uptake is also visualized within the $\mathrm{m} /$ tomND spontaneous tumor (Fig. 6). High uptake of ${ }^{18} \mathrm{~F}-(2 S, 4 R) 4$-fluoroglutamine is also apparent in other peripheral organs such as the liver, kidneys, and pancreas regions. These data are consistent with in vivo imaging studies conducted in a 9L rat xenografted animal model.

\section{In Vivo Metabolism Studies in Transgenic Mice with m/tomND Spontaneous Tumors and F344 Rats Bearing 9L Tumor Xenografts}

Metabolites of ${ }^{18} \mathrm{~F}-(2 S, 4 R) 4$-fluoroglutamine in vivo after 30 min were analyzed in both transgenic mice with $\mathrm{m} /$ tomND spontaneous tumors and F344 rats bearing 9L
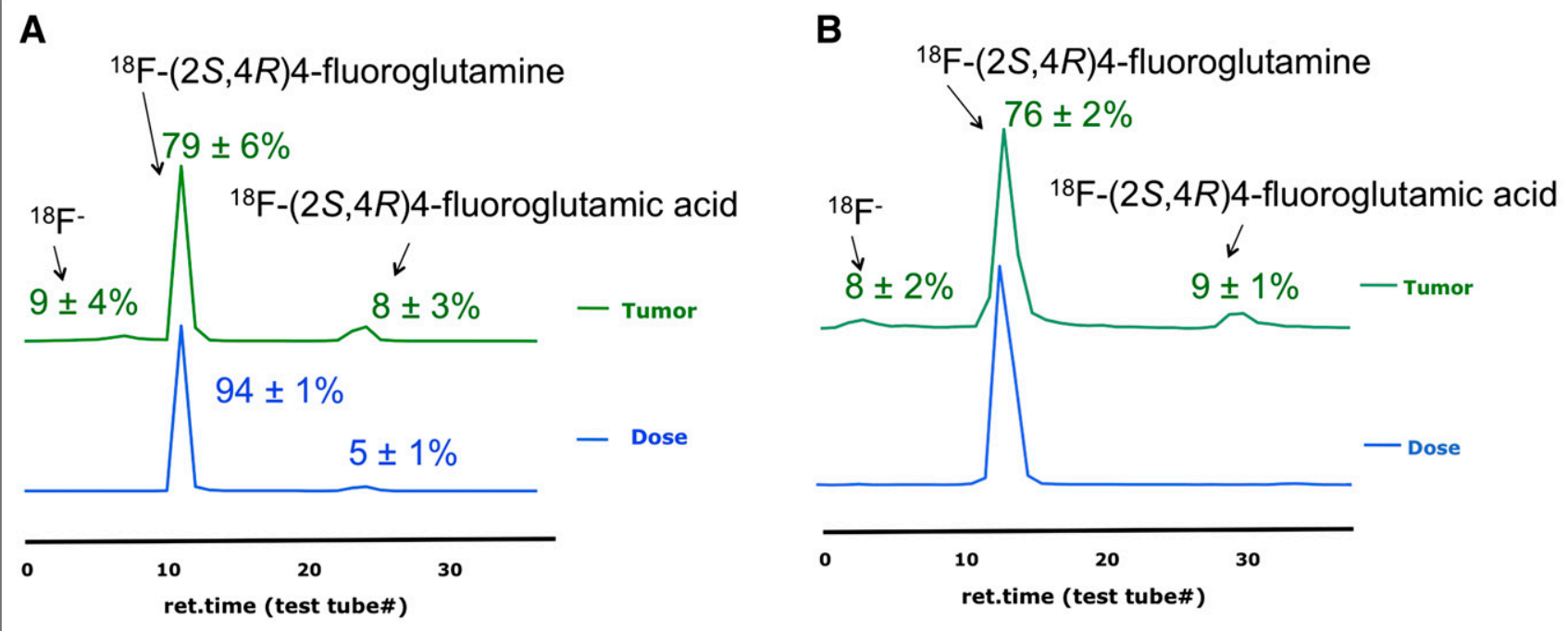

FIGURE 7. (A) Representative HPLC profiles for in vivo metabolism studies in transgenic mice bearing $\mathrm{m} /$ tomND spontaneous tumors $(n=$ 3 ) at $1 \mathrm{~h}$ after intravenous injection of ${ }^{18} \mathrm{~F}-(2 \mathrm{~S}, 4 R) 4$-fluoroglutamine. (B) Representative HPLC profiles of tumor tissue extracts (F344 rats bearing $9 \mathrm{~L}$ tumor xenografts $\left[n=2\right.$ ] at $1 \mathrm{~h}$ after intravenous injection of ${ }^{18} \mathrm{~F}-(2 S, 4 R) 4$-fluoroglutamine). ret. $=$ retention time in min. 
tumors (Fig. 7). The results are summarized in Table 3. After the tumors were homogenized and centrifuged, 29\% and $37 \%$ activity from transgenic mice and $9 \mathrm{~L}$ rats, respectively, was found to be in the pellet, probably as a result of the tracer's incorporation into macromolecule such as protein. The results demonstrated that most of the activity recovered in the supernatant remained as the parent form (79\% and $76 \%$ in transgenic mice and $9 \mathrm{~L}$ rats, respectively), and some small percentage ( $8 \%$ and $9 \%$ in transgenic mice and 9L rats, respectively) was metabolized to ${ }^{18} \mathrm{~F}-(2 S, 4 R) 4$-fluoroglutamic acid (Fig. 7). As noted in the protein-incorporation studies, ${ }^{18} \mathrm{~F}-(2 S, 4 R) 4$-fluoroglutamine showed significant in vivo incorporation to a trichloroacetic acid-precipitated fraction, suggesting that this might be a important mechanism for the trapping of this tracer in tumor tissue via glutaminolysis (Table 3).

\section{DISCUSSION}

Glutamine is the most abundant amino acid in the human body. It is an alternative energy source and a constant source of carbon and nitrogen building blocks for production of new cells $(15,16)$. It may also be possible that glutamine's role in the cell may not be limited to serving as an anabolic substrate. Glutamine also enters the TCA cycle in the mitochondria, via $\alpha$-ketoglutarate, providing reductive power and sources of adenosine triphosphate and nicotinamide adenine dinucleotide phosphate hydrogen (Fig. 1) and may play other important functions in tumor cells. Mutation and modifications of gene expression in mammalian tumor cells may constitutively change the metabolic pathways, exploiting glutamine for energy production and metabolic intermediates, essential steps for sustaining proliferation of tumor cells. Recent publications support the notion that glutamine metabolism in tumor cells also provides protection against oxidative stress, mediates new signal transduction pathways, enhances the tolerance of changes in the microenvironment, and promotes tumor growth (2, $9,31,32)$. Because of these multifaceted functions of glutamine in tumor survival and growth, there is a renewed interest in targeting glutamine metabolism as cancer therapy $(10,33)$. As such, in vivo imaging of glutamine use might be helpful in delineating the metabolic strategy tumors use. Monitoring glutaminolysis would serve as an imaging marker for tumor growth, providing critical diagnostic and prognostic information for the management of the patient with cancer. Recently, the preparation and radio- labeling of 4 isomers of 4-fluoroglutamine were reported (17). Tumor cell uptake studies (in the same tumor cells, 9L and $\left.\mathrm{SF} 188_{\mathrm{bcl}-\mathrm{xL}}\right)$ showed that 2 of the isomers, ${ }^{18} \mathrm{~F}-(2 S, 4 R)$ 4-fluoroglutamine and ${ }^{18} \mathrm{~F}-(2 S, 4 S) 4$-fluoroglutamine, displayed high uptake. The results suggest that both isomers might be associated with an increased rate of glutamine metabolism. The $2 R$ isomer showed a significantly lower tumor cell uptake, suggesting that the glutamine metabolism in the cells is highly stereoselective; a $2 S$ conformation of the 2-amino group (naturally occurring $\mathrm{L}$ amino acids are the $2 S$ isomer) is crucial for this process. Radiolabeling by a nucleophilic substitution reaction (replacing the 4- $O$-tosyl group by a naked fluoride) predominantly led to the formation of the ${ }^{18} \mathrm{~F}-(2 S, 4 R) 4$-fluoroglutamine, with higher radiolabeling yields and better stereopurities (17). For these reasons, we decided to focus on the biologic evaluation of ${ }^{18} \mathrm{~F}-(2 S, 4 R) 4$-fluoroglutamine.

In vitro tumor cell uptake studies for ${ }^{18} \mathrm{~F}-(2 S, 4 R) 4$-fluoroglutamine were performed in SF188 and SF188 $8_{\text {bcl-xL }}$ cells. The SF188 $8_{\text {bcl-xL }}$ cell line is a derivative of the SF188 cell line, engineered to constitutively express the antiapoptotic protein Bcl-xL, which improves cell survival when deprived of glutamine; both cell lines show upregulation of the myc gene (7). The Bcl-xL-modified SF188 cells are less robust; they do not survive after several passages. After unsuccessful attempts in keeping batches of SF188 $8_{\text {bcl-xL }}$ cells growing consistently, we decided to switch back to the original SF188 cells. We observed slightly different uptake and washout kinetics between these 2 cell lines; however, for practical purposes, in this paper they can be considered as equivalently upregulated c-Myc tumor cells. The glutamine tracer, ${ }^{18} \mathrm{~F}-(2 S, 4 R) 4-$ fluoroglutamine, showed rapid uptake kinetics, and the uptake can be selectively inhibited. The data suggest that glutamine uptake is driven by the myc upregulation in SF188 cells. A significant portion of the tracer is incorporated into TCA precipitated fraction, likely associated with intracellular protein or macromolecule synthesis. The protein incorporation is not observed for other ${ }^{18} \mathrm{~F}$-labeled amino acid derivatives such as 1-amino-3-fluorocyclobutyl-1-carboxylic acid (28) and $O$-(2-fluoroethyl)-1-tyrosine (34).

Animal PET studies using ${ }^{18} \mathrm{~F}-(2 S, 4 R) 4$-fluoroglutamine exhibited the expected high uptake and retention in the tumor models in rats (9L xenografted tumor) and mice (in $\mathrm{m} /$ tomND transgenic mice with spontaneous tumors).

\section{TABLE 3}

Metabolite Analysis in Spontaneous Tumors of Transgenic Mice and 9L Tumors of F344 Rats After Intravenous Injection

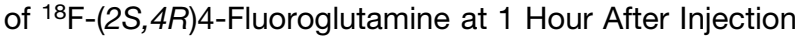

\begin{tabular}{lccc}
\hline & $\begin{array}{c}\text { Percentage parent }{ }^{18} \mathrm{~F}-(2 S, 4 R) 4- \\
\text { Animal }\end{array}$ & $\begin{array}{c}\text { Percentage metabolized }{ }^{18} \mathrm{~F}-(2 S, 4 R) 4- \\
\text { fluoroglutamine }\end{array}$ & $\begin{array}{c}\text { Percentage protein } \\
\text { pellet activity }\end{array}$ \\
\hline Transgenic mice $(n=3)$ & $79 \pm 6.0$ & $8.0 \pm 3.0$ & $29 \pm 6.0$ \\
F344 rats $(n=2)$ & $76 \pm 2.0$ & $9.0 \pm 1.0$ & $37 \pm 20$ \\
\hline
\end{tabular}


The 9L xenografted tumor model is well known in the literature for studying novel tumor imaging agents $(18,30,35)$. The imaging studies in this rat tumor model clearly confirmed that the agent is highly sequestered in tumor tissue, and the trapping was highly persistent, suggesting that the tracer was taken up and incorporated into protein, other cytosol macromolecules, and the large intracellular glutamic acid pool. However, the linkage between c-Myc and quantitative PET of glutaminolysis is not yet fully established. Genetically engineered mouse tumor models are highly useful to study tumor biology (36). We decided to use $\mathrm{m} /$ tomND transgenic mice with spontaneous mammary gland tumors as a model for testing, because they are closer to the native than the xenografted tumor model. We used HER2/neu expression controlled by doxycycline administered through the drinking water to develop a mouse tumor model (m/tomND transgenic mice) in which $m y c$ expression could be manipulated $(37,38)$. These mice spontaneously grow mammary gland tumors in a few weeks, and myc expression in the tumors was upregulated by 2-fold under doxycycline-triggered myc upregualtion (Alveraz and Chodosh, unpublished data, 2011). Currently, efforts are being made to correlate the polymerase chain reaction-measured myc gene expression to ${ }^{18} \mathrm{~F}-(2 S, 4 R) 4-$ fluoroglutamine tumor uptake in this transgenic model. One obstacle not easy to overcome is that the size of the tumor varies under different c-Myc gene expression levels; thus, obtaining quantitative data of tumor uptake by animal PET studies is difficult to accomplish. Correction for tumor size might alleviate some of the variations, but the quantitative correlation of c-Myc gene expression and glutamine uptake may be a challenge not yet easily resolved.

\section{CONCLUSION}

This paper demonstrates that in vitro tumor cell uptake of ${ }^{18} \mathrm{~F}-(2 S, 4 R) 4$-fluoroglutamine is higher than uptake of ${ }^{3} \mathrm{H}-$ L-glutamine and ${ }^{18}$ F-FDG. In vivo PET studies showed significant tumor uptake and trapping inside tumor tissue in 2 different tumor models. The results support the feasibility of PET for the study of a fundamental change in tumor metabolism-high-rate glutaminolysis.

\section{DISCLOSURE STATEMENT}

The costs of publication of this article were defrayed in part by the payment of page charges. Therefore, and solely to indicate this fact, this article is hereby marked "advertisement" in accordance with 18 USC section 1734.

\section{ACKNOWLEDGMENTS}

We thank Dr. Chaitanya Divgi and Hans Seidel for providing helpful discussions. This work was supported in part by a grant from Stand-Up 2 Cancer (SU2C), a medical scientist training grant, and a grant from the Cancer Research Institute. No other potential conflict of interest relevant to this article was reported.

\section{REFERENCES}

1. Warburg O. On the origin of cancer cells. Science. 1956;123:309-314.

2. Dang C. Rethinking the Warburg effect with myc micromanaging glutamine metabolism. Cancer Res. 2010;70:859-862.

3. Vander Heiden M, Cantley L, Thompson C. Understanding the Warburg effect: the metabolic requirements of cell proliferation. Science. 2009;324:10291033.

4. Gillies RJ, Robey I, Gatenby RA. Causes and consequences of increased glucose metabolism of cancers. J Nucl Med. 2008;49(suppl 2):24S-42S.

5. Gambhir SS. Molecular imaging of cancer: from molecules to humans-introduction. J Nucl Med. 2008;49(suppl 2):1S-4S

6. Robey IF, Stephen RM, Brown KS, Baggett BK, Gatenby RA, Gillies RJ. Regulation of the Warburg effect in early-passage breast cancer cells. Neoplasia. 2008;10:745-756.

7. Wise D, DeBerardinis R, Mancuso A, et al. Myc regulates a transcriptional program that stimulates mitochondrial glutaminolysis and leads to glutamine addiction. Proc Natl Acad Sci USA. 2008;105:18782-18787.

8. Albihn A, Johnsen J, Henriksson M. Myc in oncogenesis and as a target for cancer therapies. Adv Cancer Res. 2010;107:163-224.

9. Dang CV. Glutaminolysis: supplying carbon or nitrogen or both for cancer cells? Cell Cycle. 2010;9:3884-3886.

10. Dang CV, Hamaker M, Sun P, Le A, Gao P. Therapeutic targeting of cancer cell metabolism. J Mol Med. 2011;89:205-212.

11. Wellen KE, Thompson CB. Cellular metabolic stress: considering how cells respond to nutrient excess. Mol Cell. 2010;40:323-332.

12. Gao P, Tchernyshyov I, Chang T, et al. C-myc suppression of mir-23a/b enhances mitochondrial glutaminase expression and glutamine metabolism. Nature. 2009; 458:762-765.

13. Thompson C. Metabolic enzymes as oncogenes or tumor suppressors. $N$ Engl J Med. 2009;360:813-815.

14. Cheng T, Sudderth J, Yang C, et al. Pyruvate carboxylase is required for glutamineindependent growth of tumor cells. Proc Natl Acad Sci USA. 2011;108: 8674-8679.

15. Rajagopalan KN, Deberardinis RJ. Role of glutamine in cancer: therapeutic and imaging implications. J Nucl Med. 2011;52:1005-1008.

16. Shanware NP, Mullen AR, DeBerardinis RJ, Abraham RT. Glutamine: pleiotropic roles in tumor growth and stress resistance. J Mol Med. 2011;89:229-236.

17. Qu W, Zha Z, Ploessl K, et al. Synthesis of optically pure 4-fluoro-glutamines as potential metabolic imaging agents for tumors. J Am Chem Soc. 2011;133:11221133 .

18. Yu W, Williams L, Camp V, Olson J, Goodman M. Synthesis and biological evaluation of anti-1-amino-2-[18F]fluoro-cyclobutyl-1-carboxylic acid (anti-2[18F]FACBC) in rat 91 gliosarcoma. Bioorg Med Chem Lett. 2010;20:2140-2143.

19. Martarello L, McConathy J, Camp VM, et al. Synthesis of syn- and anti1-amino-3-[18F]fluoromethyl-cyclobutane-1-carboxylic acid (FMACBC), potential pet ligands for tumor detection. J Med Chem. 2002;45:2250-2259.

20. Schuster DM, Savir-Baruch B, Nieh PT, et al. Detection of recurrent prostate carcinoma with anti-1-amino-3-18F-fluorocyclobutane-1-carboxylic acid PET/ CT and ${ }^{111}$ In-capromab pendetide SPECT/CT. Radiology. 2011;259:852-861.

21. McConathy J, Goodman Mark M. Non-natural amino acids for tumor imaging using positron emission tomography and single photon emission computed tomography. Cancer Metastasis Rev. 2008;27:555-573.

22. Wasa M, Wang HS, Okada A. Characterization of L-glutamine transport by a human neuroblastoma cell line. Am J Physiol Cell Physiol. 2002;282:C1246-C1253.

23. McGivan JD, Bungard CI. The transport of glutamine into mammalian cells. Front Biosci. 2007;12:874-882.

24. Nakanishi T, Sugawara M, Huang W, et al. Structure, function, and tissue expression pattern of human sn2, a subtype of the amino acid transport system $\mathrm{n}$. Biochem Biophys Res Commun. 2001;281:1343-1348.

25. Bodoy S, Martin L, Zorzano A, Palacin M, Estevez R, Bertran J. Identification of lat4, a novel amino acid transporter with system 1 activity. J Biol Chem. 2005; 280:12002-12011.

26. Kekuda R, Prasad PD, Fei YJ, et al. Cloning of the sodium-dependent, broadscope, neutral amino acid transporter Bo from a human placental choriocarcinoma cell line. J Biol Chem. 1996;271:18657-18661.

27. Fuchs BC, Bode BP. Amino acid transporters asct 2 and lat 1 in cancer: partners in crime? Semin Cancer Biol. 2005;15:254-266.

28. Okudaira H, Shikano N, Nishii R, et al. Putative transport mechanism and intracellular fate of trans-1-amino-3-18F-fluorocyclobutanecarboxylic acid in human prostate cancer. J Nucl Med. 2011;52:822-829.

29. Esslinger CS, Cybulski KA, Rhoderick JF. Ngamma-aryl glutamine analogues as probes of the asct 2 neutral amino acid transporter binding site. Bioorg Med Chem. 2005;13:1111-1118. 
30. Lee TS, Ahn SH, Moon BS, et al. Comparison of 18F-FDG, 18F-FET and 18FFLT for differentiation between tumor and inflammation in rats. Nucl Med Biol. 2009;36:681-686.

31. Weinberg F, Chandel N. Mitochondrial metabolism and cancer. Ann NY Acad Sci. 2009;1177:66-73.

32. DeBerardinis R, Cheng T. Q's next: the diverse functions of glutamine in metabolism, cell biology and cancer. Oncogene. 2010;29:313-324.

33. Dang C, Le A, Gao P. Myc-induced cancer cell energy metabolism and therapeutic opportunities. Clin Cancer Res. 2009;15:6479-6483.

34. Wester H, Herz M, Weber W, et al. Synthesis and radiopharmacology of O(2-[18F]fluoroethyl)-1-tyrosine for tumor imaging. J Nucl Med. 1999;40: $205-212$.
35. Yu W, Williams L, Camp V, Malveaux E, Olson J, Goodman M. Stereoselective synthesis and biological evaluation of syn-1-amino-3-[18F]fluorocyclobutyl-1carboxylic acid as a potential positron emission tomography brain tumor imaging agent. Bioorg Med Chem. 2009;17:1982-1990.

36. Blakely CM, Sintasath L, D'Cruz CM, et al. Developmental stage determines the effects of myc in the mammary epithelium. Development. 2005;132:1147-1160.

37. Yeh ES, Yang TW, Jung JJ, Gardner HP, Cardiff RD, Chodosh LA. Hunk is required for HER2/neu-induced mammary tumorigenesis. J Clin Invest. 2011; 121:866-879.

38. Schoenherr RM, Kelly-Spratt KS, Lin C, et al. Proteome and transcriptome profiles of a her2/neu-driven mouse model of breast cancer. Proteomics Clin Appl. 2011;5:179-188. 